\title{
Feasibility and effect of para-right bundle branch pacing in patients with atrial fibrillation and complete atrioventricular block
}

\author{
You-You Du, Rui Yao, Qing-Hua Chen, Luo-Sha Zhao, Ling Li \\ Department of Cardiology, the First Affiliated Hospital of Zhengzhou University, Zhengzhou, China
}

\begin{abstract}
Background: Chronic right ventricular apex (RVA) pacing can induce negative clinical effects. The aim of the present study was to compare RVA pacing with para-right bundle branch (para-RBB) pacing in terms of electrocardiogram (ECG) and echocardiographic (ECHO) features.

Methods: Forty-one consecutive persistent atrial fibrillation patients with an indication for permanent pacing treatment due to complete atrioventricular block were randomly assigned to receive a screw-in lead either in the $R V A(n=22)$ or at the para-RBB $(n=19)$. Para-RBB pacing leads were located according to the RBB potential recorded by electrophysiology catheter. ECG was recorded before and after implantation. All patients underwent the pacemaker programming at 1 day, 6 months, 12 months and 24 months after implantation. ECHO examination was performed during follow-up at 6, 12 and 24 months after implantation to assess the heart function and synchronism.
\end{abstract}

Results: There was no significant difference in pacing lead parameters between para-RBB pacing group and RVA pacing group. Compared with RVA pacing group, the para-RBB pacing group obtained a narrower QRS complex, more synchronic ventricular systole, and less negative effect on heart function $(p<0.05)$.

Conclusions: Para-RBB pacing has potential clinical benefits and may be a physiological pacing site. (Cardiol J 2015; 22, 3: 233-240)

Key words: cardiac pacing, atrioventricular block, atrial fibrillation, right bundle branch

\section{Introduction}

Permanent cardiac pacing is the most optimal treatment for patient with high-degree atrioventricular block (AVB) and symptomatic sick sinus syndrome (SSS) [1]. Traditionally, the right ventricular (RV) pacing leads have been fixed in the RV apex (RVA) [2]. Pacing from the RVA triggers a special wave of depolarization, which induces an aberrant ventricular activation and an elongation of ventricular activation time with consequent postponed activation of the left ventricular lateral wall [3]. Prolonged pacing from the RVA is associated with progressive left ventricular dysfunction, exacerbation of heart failure, atrial fibrillation, and an increased morbidity and mortality [4]. New pacemaker algorithms reducing ventricular pacing can minimize the percentage of ventricular pacing in patients with SSS, but not in patients with high-grade AVB [5].These observations have led to a growing interest in searching for alternate ventricular pacing sites in order to achieve a less

Address for correspondence: You-You Du, MD, Department of Cardiology, the First Affiliated Hospital of Zhengzhou University, No. 1 Jianshe East Road, Zhengzhou, 450052, China, tel: +86 13526413832, e-mail: duyouyou_008@163.com 
eccentric, more physiologic pattern of ventricular activation.

So far, the alternate pacing sites have included the RV outflow tract (RVOT), RV inflow tract, His-bundle, para-Hisian and biventricle. Theoretically, direct His-bundle pacing, para-Hisian pacing and biventricular pacing are the ideal sites, but they are time-consuming and difficult procedures. Up to now, the most studied of these selective sites has been the RVOT with increasing focus on the septal aspect of this structure. However, the true RVOT septal pacing has until recently been difficult to consistently achieve [6].

Studies indicate that the distance of the pacing site in the RV from the His-Purkinje System contributes the length of the QRS interval and substantial changes of the activation pattern [7]. Mapping data showed that the higher the RVOT pacing position, the farther it is from His-Purkinje System, and the ideal position seems to be pacing from the mid-septum, where the earliest endocardial signal from the right bundle branch $(\mathrm{RBB}) \mathrm{can}$ be observed [8]. On this basis, we hypothesized that para-RBB in the mid-septum could become a new elective site for ventricular physiological pacing. The aims of this study were to evaluate the feasibility and security of para-RBB pacing, and to compare the long-term effect of para-RBB pacing and RVA pacing on ventricular function and synchronism.

\section{Methods}

\section{Study patients}

The inclusion criteria were: (1) the existence of persistent atrial fibrillation to rule out any latent hemodynamic interference of the atrial systole and the modulation of atrioventricular delay in patients with dual chamber pacing; (2) the existence of complete AVB with torpid ventricular rate to ensure permanent and complete ventricular pacing; (3) the existence of a junctional escape rhythm to make the mapping RBB potential possible.

Patients with significant valvular heart disease, serious coronary artery disease, congestive heart failure, frequent ventricular arrhythmia, Electrocardiogram (ECG) indications for bundle branch block and the left ventricular ejection fraction $(\mathrm{LVEF})<50 \%$ were excluded from the study.

All cases gave their informed consent, and the study protocol was approved by the Institutional Ethics Committee of the First Affiliated Hospital of Zhengzhou University.

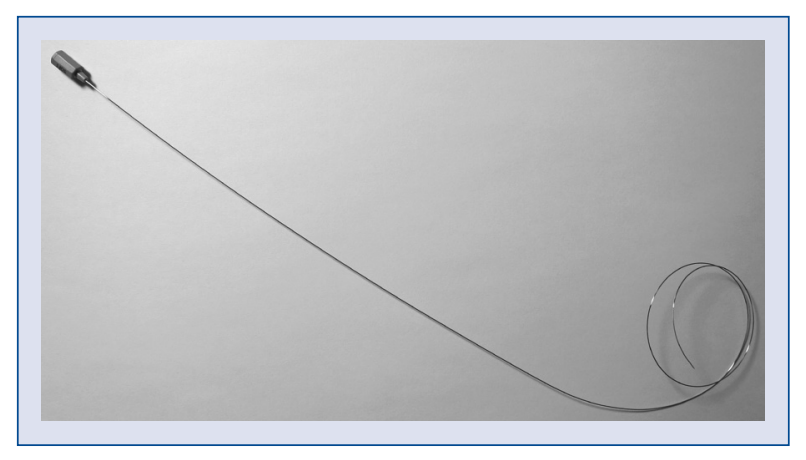

Figure 1. The stylet used for para-right bundle branch lead implantation.

\section{Implantation of ventricular pacing leads}

All patients underwent implantation of single-chamber (VVI/VVIR) pacemakers using the active fixation leads (St. Jude $1888 \mathrm{TC}-58 \mathrm{~cm}$ ) under local anesthesia. Patients were randomly divided into two groups: RVA pacing group and para-RBB pacing group.

In the RVA pacing group, the ventricular pacing leads were placed in the RVA. In the para-RBB pacing group, the pacing leads were positioned near the RBB in the mid-septum. During the implantation of para-RBB pacing leads, a bipolar diagnostic electrophysiology (EP) catheter with $5 \mathrm{~mm}$ interelectrode spacing was introduced via the right femoral vein and was advanced to the septal aspect of RVOT to locate the RBB guided by the RBB potential. Via the left subclavian vein, the pacing leads were positioned close to the RBB guided by the RBB potential recorded by the EP catheter. In order to achieve para-RBB pacing, a ring-shaped stylet was used as an introducer tool (Fig. 1). During the operation, the pacing leads with ring-shaped stylet could easily pass tricuspid annulus, and be send to the RVOT. With slight withdrawal of the stylet, the head of electrode would directly point to the septum. By fine adjustment, the pacing leads could be positioned near the RBB.

We defined para-RBB pacing in accordance with both of the following: (1) the pacing leads were confirmed to be close to the tip of EP catheter mapping RBB potential by fluoroscopy (Fig. 2); (2) QRS shortening at high pacing outputs because of RBB captured (Fig. 3).

In addition, the test results of each pacing lead parameter should be consistent with the following criteria: first, ventricular pacing threshold $<1.5 \mathrm{~V} /$ $/ 0.5 \mathrm{~ms}$, $\mathrm{R}$ wave sensing $>5.0 \mathrm{mV}$ and pacing lead 


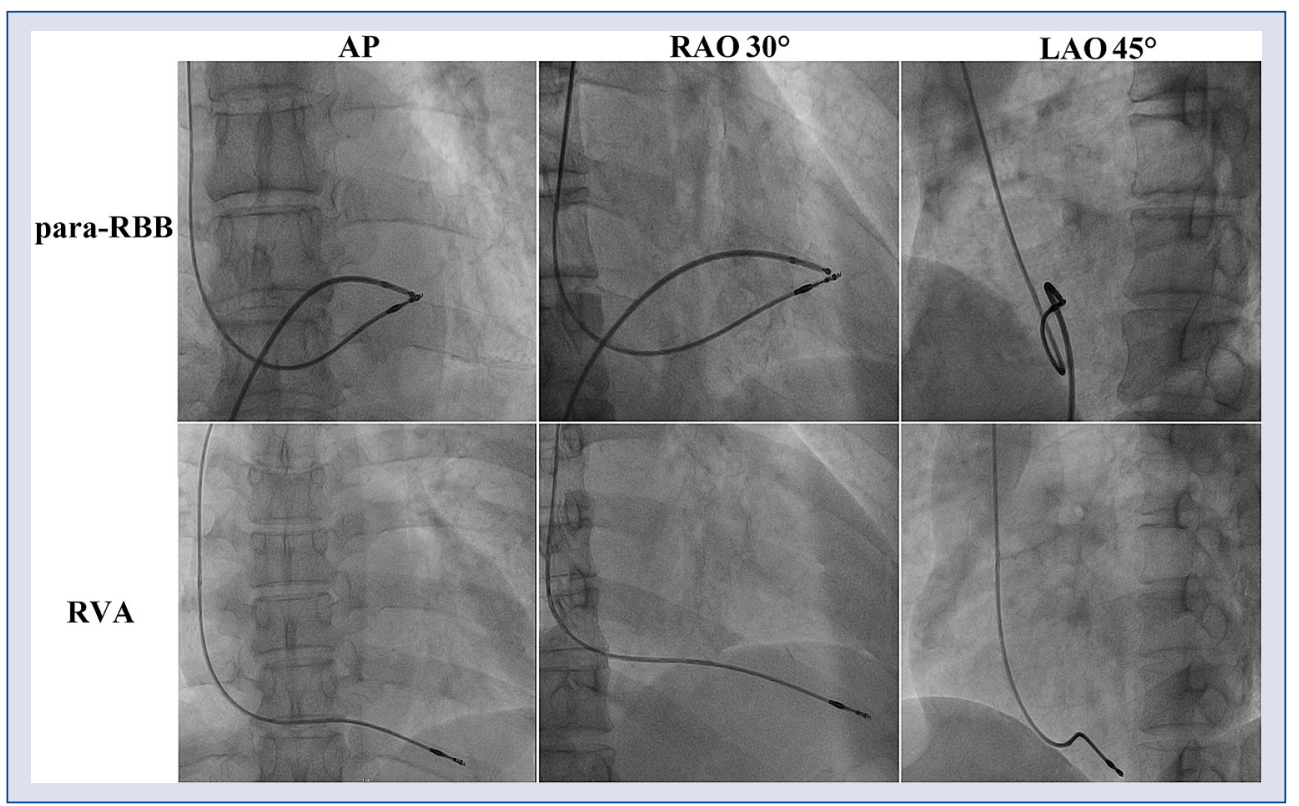

Figure 2. Image positioning of para-right bundle branch (para-RBB) pacing and right ventricular apex (RVA) pacing. The tip of pacing lead of para-RBB pacing is close to the first pole of mapping electrode; AP — anterior posterior; RAO — right anterior oblique; LAO — left anterior oblique.

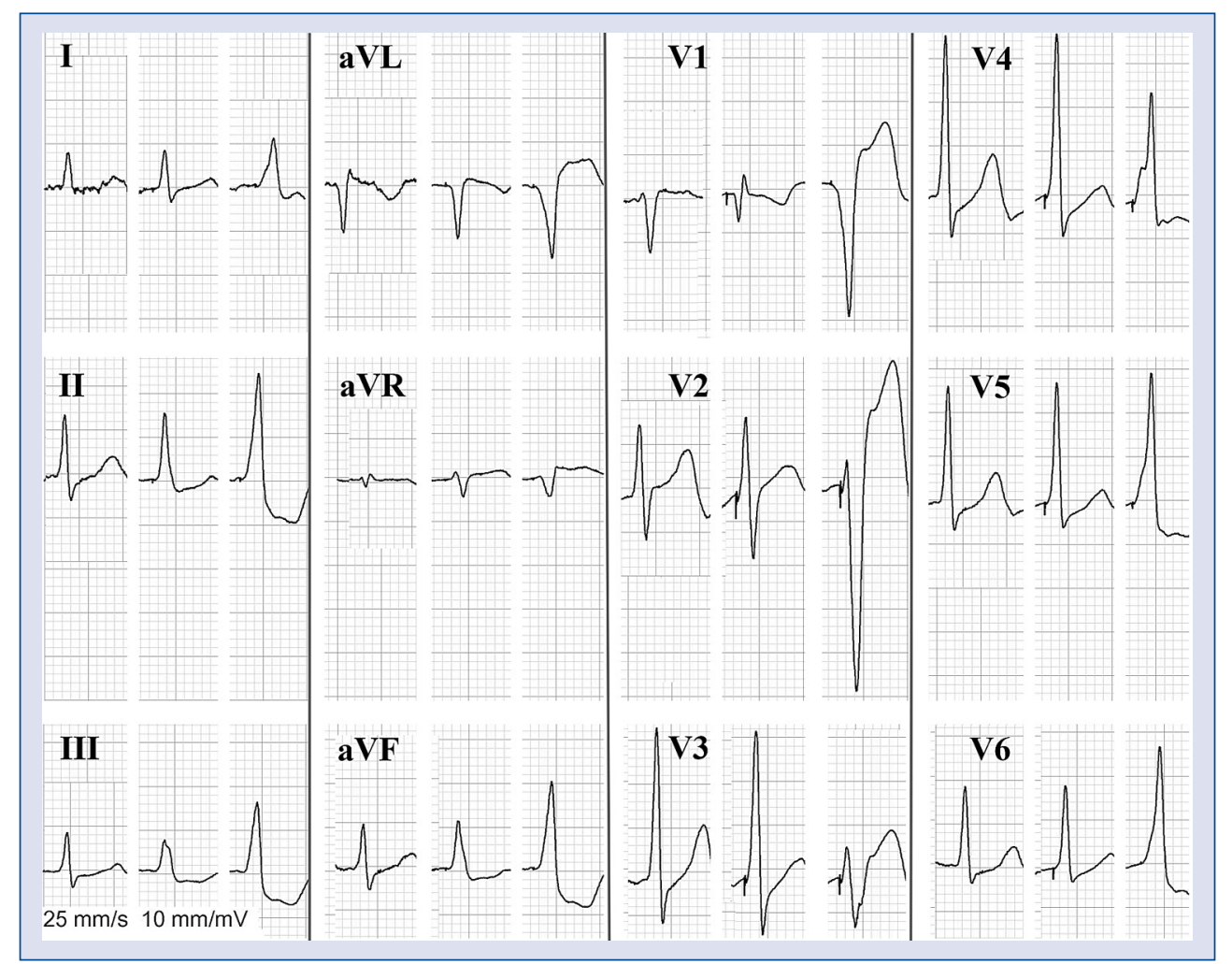

Figure 3. The QRS complex of capture and non-capture of right bundle branch (RBB). In 12 leads electrocardiogram, the first QRS complex is the inherent QRS complex of the patient. The second is the QRS complex of the capture of RBB. The third is the QRS complex of ventricular capture and non-capture of RBB. 
impedance was $300 \sim 1,000 \Omega$, and second, RBB capture threshold $<3.0 \mathrm{~V} / 1 \mathrm{~ms}$.

\section{Study protocol}

Pacemaker programming was performed at 1 day, 6,12 and 24 months after implantation. The basic pacing rate of pacemaker was set at $60 \mathrm{bpm}$. All patients underwent echocardiographic (ECHO) examination during follow-up at 6,12 and 24 months after pacemaker implantation to assess the heart function and synchronism.

The ECHO studies were performed by the same operator with a GE Vivid 7 ultrasound system as previously described [9]. The left ventricular end-diastolic diameter (LVEDD) was measured using M-mode by the apical 4-chamber view. In addition, the LVEF was calculated with the modified Simpson's method. In the parasternal short axis, the sequence between the contractions of septal and posterior walls at the level of the papillary muscle was measured as the septal-to-posterior wall motion delay (SPWMD) [9]. The intervals between the onset of the QRS complex to the initiation of ejection at the pulmonary and the aortic valve levels were determined as the pulmonary pre-ejection interval (PPEI) and the aortic preejection interval (APEI), respectively. The difference between APEI and PPEI was defined as the interventricular mechanical delay (IVMD).

In order to measure the duration of paced QRS complex, the standard 12-channel surface ECG at $25 \mathrm{~mm} / \mathrm{s}$ speed was recorded before and after the operation, and the QRS complex duration was measured for comparative analyses.

\section{Statistical analysis}

Continuous variables of normal distribution are described as mean \pm standard deviation. Categorical data are described as frequencies and percentages. Comparisons between the two groups were performed by Student's t-test for normally distributed continuous variables and by the Pearson $\chi^{2}$ test for categorical variables. The pared Student's t test was used to compare the change in variables in each group. $\mathrm{P}$ values $<0.05$ were considered statistically significant. All statistical analyses were performed with SPSS Version 21.

\section{Results}

\section{Patient characteristics}

From March 2009 to December 2011, 41 patients were enrolled in this study: 19 patients were randomized to receive para-RBB pacing, and 22 patients to RVA pacing. At baseline, there were no significant differences between two groups. All the data were summarized in Table 1 .

\section{Implantation procedure}

Pacing systems were successfully implanted in all patients, and no complications or lead dislodgment was observed during the whole study. During the RV pacing leads implantation, there were significant differences both in testing site frequency and in X-ray exposure time between para-RBB pacing group and RVA pacing group; testing site frequency: $3.37 \pm 0.90$ vs. $1.55 \pm 0.74, \mathrm{p}<0.001$, $\mathrm{X}$-ray exposure time [min]: $5.85 \pm 0.90$ vs. $2.29 \pm 0.64, \mathrm{p}<0.001$.

\section{Pacing measurements}

Chronic pacing lead parameters, including perception, impedance and threshold of ventricular pacing, were stable and did not differ significantly between the two groups. In addition, there was no significant difference in the percent of ventricular pacing between the two groups. All these data were summarized in Table 2.

\section{Analysis of QRS complex}

There was no difference in QRS duration of intrinsic rhythm between the two groups (Table 1). After implantation, the QRS duration was significantly longer in RVA pacing group than in para-RBB pacing group (149.82 $\pm 8.98 \mathrm{~ms}$ vs. $126.00 \pm 5.21 \mathrm{~ms}$, $\mathrm{p}<0.001$ ) (Fig. 4). Compared with para-RBB pacing group, the electrical axis of QRS in RVA pacing group was significantly left-deviated $\left(-63.6 \pm 7.6^{\circ}\right.$ vs. $\left.52.4 \pm 7.7^{\circ}, \mathrm{p}<0.001\right)$.

\section{Analysis of left ventricular performance and synchronism}

There were no significant differences in LVEF, LVEDD, IVMD and SPVMD at baseline between the two groups (Table 1). In RVA pacing group, compared with baseline, LVEF at 24 months after implantation was decreased significantly $(53.68 \pm$ $\pm 4.41 \%$ vs. $58.18 \pm 3.83 \%, \mathrm{p}<0.001$ ), and LVEDD at 12 and 24 months after implantation was increased significantly (12 months: $53.09 \pm 3.2 \mathrm{~mm}$ vs. $49.95 \pm 3.93 \mathrm{~mm}, \mathrm{p}<0.001 ; 24$ months: $55.59 \pm$ $\pm 3.25 \mathrm{~mm}$ vs. $49.95 \pm 3.93 \mathrm{~mm}, \mathrm{p}<0.001)$ (Fig. 5A, B). There was significant difference in LVEF and LVEDD between RVA pacing group and para-RBB pacing group at 24 months after implantation (LVEF: $53.68 \pm 4.41 \%$ vs. $57.74 \pm 4.09 \%$, $\mathrm{p}=0.004$; LVEDD: $55.59 \pm 3.25 \mathrm{~mm}$ vs. $52.00 \pm$ 
Table 1. Basic clinical, echocardiographic, and other important patient characteristics.

\begin{tabular}{|c|c|c|c|}
\hline Variables & RVA (n = 22) & Para-RBB (n = 19) & $\mathbf{P}$ \\
\hline Age [years] & $51 \pm 9$ & $54 \pm 11$ & 0.344 \\
\hline Male sex & $8(36.4 \%)$ & $7(36.8 \%)$ & 0.975 \\
\hline LVEDD [mm] & $49.95 \pm 3.93$ & $51.63 \pm 3.93$ & 0.181 \\
\hline LVEF [\%] & $57.77 \pm 4.24$ & $58.37 \pm 4.81$ & 0.676 \\
\hline IVMD [ms] & $12.84 \pm 2.44$ & $13.38 \pm 2.72$ & 0.508 \\
\hline SPVMD [ms] & $33.59 \pm 3.83$ & $32.42 \pm 4.13$ & 0.352 \\
\hline QRS duration [ms] & $88.82 \pm 6.26$ & $88.11 \pm 6.64$ & 0.726 \\
\hline Hypertension & $12(54.5 \%)$ & $10(52.6 \%)$ & 0.902 \\
\hline Coronary artery disease & $8(36.4 \%)$ & $7(36.8 \%)$ & 0.975 \\
\hline Diabetes mellitus & $6(27.3 \%)$ & $5(26.3 \%)$ & 0.922 \\
\hline Heart rate & $40.4 \pm 3.7$ & $39.9 \pm 3.9$ & 0.701 \\
\hline Pacemaker mode: & & & 0.699 \\
\hline VVI & $15(68.2 \%)$ & $14(73.7 \%)$ & \\
\hline VVIR & $7(31.8 \%)$ & $5(26.3 \%)$ & \\
\hline Mitral VR: & & & 0.887 \\
\hline Mild & $7(31.8 \%)$ & $7(36.8 \%)$ & \\
\hline Moderate & $6(27.3 \%)$ & $4(21.1 \%)$ & \\
\hline Normal & $9(40.9 \%)$ & $8(42.1 \%)$ & \\
\hline Tricuspid VR: & & & 0.939 \\
\hline Mild & $8(36.4 \%)$ & $6(31.6 \%)$ & \\
\hline Moderate & $5(22.7 \%)$ & $5(26.3 \%)$ & \\
\hline Normal & $9(40.9 \%)$ & $8(42.1 \%)$ & \\
\hline Diuretic & $15(68.2 \%)$ & $14(73.7 \%)$ & 0.699 \\
\hline ACEI/ARB & $13(59.1 \%)$ & $12(63.2 \%)$ & 0.790 \\
\hline Beta-blocker & $8(36.4 \%)$ & $8(42.1 \%)$ & 0.707 \\
\hline Calcium channel blocker & $10(45.5 \%)$ & $10(52.6 \%)$ & 0.647 \\
\hline
\end{tabular}

RVA — right ventricular apex; Para-RBB — para-right bundle branch; LVEDD — left ventricular end-diastolic diameter; LVEF — left ventricular ejection fraction; IVMD - interventricular mechanical delay; SPWMD — septal-to-posterior wall motion delay; VR — valve regurgitation; $\mathrm{ACEI}$ - angiotensin converting enzyme inhibitor; ARB - angiotensin receptor blocker

Table 2. Pacing lead parameters measurements.

\begin{tabular}{|c|c|c|c|c|}
\hline \multirow[t]{2}{*}{ Variables } & \multicolumn{4}{|c|}{ Duration after pacemaker implantation } \\
\hline & 1 day & 6 months & 12 months & 24 months \\
\hline \multicolumn{5}{|l|}{ RVA pacing $(n=22)$} \\
\hline Pacing percent [\%] & $98.0 \pm 1.3$ & $98.1 \pm 1.0$ & $98.1 \pm 0.9$ & $98.3 \pm 0.8$ \\
\hline Threshold [V] & $0.68 \pm 0.28$ & $0.75 \pm 0.22$ & $0.80 \pm 0.20$ & $0.78 \pm 0.16$ \\
\hline Perception [mV] & $9.50 \pm 2.90$ & $9.40 \pm 2.52$ & $9.25 \pm 2.06$ & $9.32 \pm 1.98$ \\
\hline Impedance $[\Omega]$ & $615 \pm 127$ & $629 \pm 107$ & $634 \pm 92$ & $637 \pm 87$ \\
\hline \multicolumn{5}{|c|}{ Para-RBB pacing ( $n=19$ ) } \\
\hline Pacing percent [\%] & $97.7 \pm 1.1$ & $98.2 \pm 1.1$ & $98.2 \pm 0.9$ & $98.5 \pm 0.7$ \\
\hline Threshold [V] & $0.70 \pm 0.31$ & $0.78 \pm 0.30$ & $0.82 \pm 0.20$ & $0.82 \pm 0.16$ \\
\hline Perception [mV] & $8.29 \pm 2.09$ & $8.21 \pm 1.87$ & $8.27 \pm 1.38$ & $8.29 \pm 1.28$ \\
\hline Impedance $[\Omega]$ & $592 \pm 111$ & $579 \pm 102$ & $589 \pm 108$ & $592 \pm 101$ \\
\hline
\end{tabular}

RVA — right ventricular apex; Para-RBB — para-right bundle branch 


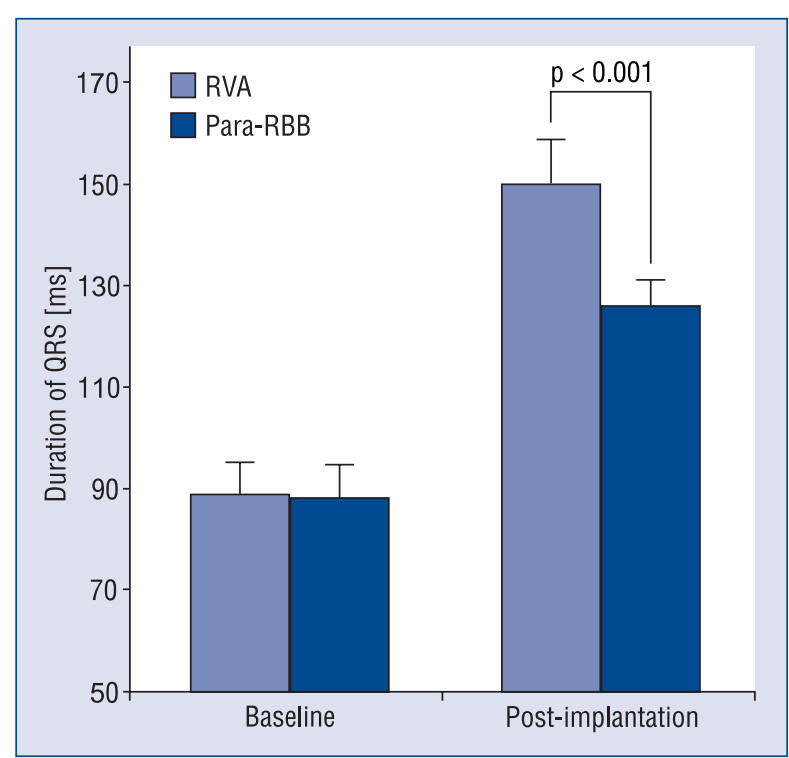

Figure 4. Difference in QRS complex duration between right ventricular apex (RVA) pacing group and para-right bundle branch (para-RBB) pacing group.

$\pm 3.92 \mathrm{~mm}, \mathrm{p}=0.003$ ) (Fig. 5A, B). Compared with baseline, the IVMD and SPWMD of the two groups after implantation were significantly increased $(\mathrm{p}<0.05)$. And during the follow-up, there was a significant difference between the two groups in the IVMD and SPWMD ( $<$ 0.05) (Fig. 5C, D).

\section{Discussion}

The optimal ventricular pacing site should simulate the normal activation and synchronism of ventricular activation observed with an undamaged conduction system [4]. In the present study, we compared the clinical effect of paraRBB pacing and RVA pacing on the ventricular structure and function and found that compared with pacing from RVA, pacing from para-RBB is more physiological and could obtain more clinical advantage. To our knowledge, it is the first time up to now to report the new physiological pacing site of para-RBB.

The traditional RVA pacing allows easy endocardial implantation of pacing leads while providing the stable and reliable pacing parameters. In spite of these, RVA pacing triggers an abnormal electrical activation pattern, and results in asynchronous ventricular contraction and relaxation. Recently, many studies have demonstrated that permanent RVA pacing was associated with negative clinical effects [10]. Similarly, our study confirmed that the
LVEF and LVEDD deteriorated during long-term RVA pacing.

Activation of the ventricular myocardium by pacing at His-Purkinye system could cause a synchronous activation. Recently, small samples studies have suggested direct His-bundle pacing $[11,12]$ and para-His pacing $[5,13]$ as more physiological pacing sites maintaining the normal ventricular activation pattern. Theoretically, these are the ideal physiological pacing sites. However, due to the specificity of the anatomic structure of His-bundle, pacing at these sites has certain limitations. First, the actual operation is relatively complex and the implantation technique is challenging. Second, autopsy analysis of the implantation site of direct His-bundle pacing has shown unequivocally that the pacing lead is implanted on the atrial side of the tricuspid annulus [14]. In fact, the implantation lead on this site had a poor sensed ventricle signal. Therefore, a backup lead should be inserted into $\mathrm{RV}$ to ensure an adequate ventricular sensing [14]. Third, for patients of AVB with the blocking site at the distal His-bundle, direct His-bundle pacing is not a proper choice. Fourth, His-bundle penetrates the atrioventricular ring, extends along the lower edge of the membranous ventricular septum [15]. The pacing lead implanted into this site might lead to the perforation of the membranous ventricular septum.

In the 1970s, Durrer's study [16] identified the earliest ventricular activation in the RVOT, and pacing at this region might obtain physiological ventricular activation pattern. Subsequently, more and more studies of non-RVA pacing have centralized in this region. However, because of the poor description of RVOT and the lacking of consistent anatomic designation, the pacing sites at RVOT in the published studies include a variety of pacing sites indeed, such as true outflow tract, mid-septum and anterior region above the apex. Therefore, the acute and chronic studies of RVOT pacing have produced conflicting results [17].

The mapping data of Kappos's investigation has indicated that the mid-septum seems to be the ideal site for RV pacing, where the earliest endocardial signal from the RBB can be observed [8]. In addition, Muto' et al. [2] study suggests that pacing from RV mid-septum is more physiological than pacing from RVA. Based on these findings, our study used a bipolar electrode to guide the pacing lead to be fixed near the RBB in the mid-septum by mapping the RBB potential. It is exhilarating that the activation can capture the 


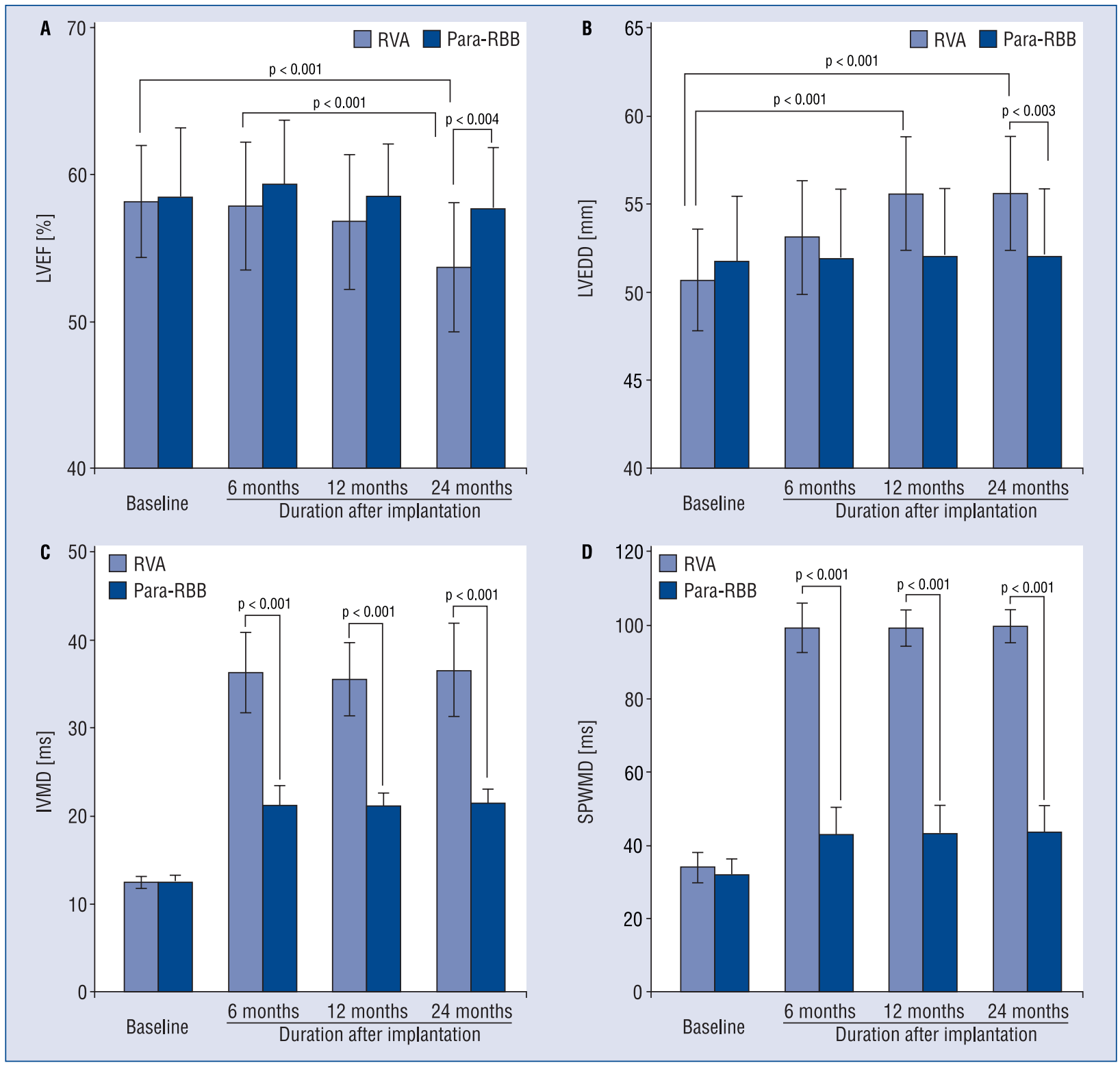

Figure 5. A-D. Difference in ventricular function and synchronism between right ventricular apex (RVA) pacing group and para-right bundle branch (Para-RBB) pacing group; LVEF — left ventricular ejection fraction; LVEDD — left ventricular end-diastolic diameter; IVMD — interventricular mechanical delay; SPWMD - septal-to-posterior wall motion delay.

RBB by fine-tuning the distance between pacing lead and RBB. Previous study indicated that conduction fibers in His-bundle and proximal bundle branches were freely interconnected transversely, and this was unequivocal for RBB [18]. On capturing the RBB, the activation signal could advance rapidly along the $\mathrm{RBB}$ and antidromically activate his-bundle and left bundle branch. In this way, a relatively physiological activation pattern of ventricle was followed. In our study, compared with pacing from RVA, pacing from para-RBB induced a shorter QRS complex duration and a more synchronous ventricular activation.

\section{Limitations of the study}

There are several limitations of the present study. First, there was a small number of patients included. Second, follow-up period was short. Third, the approach of implanting para-RBB leads requires repeated adjustments of implantation site and long X-ray exposure time. Therefore, the implantation technique should be improved. 


\section{Conclusions}

To sum up, para-RBB pacing is a special site pacing in mid-septum. Pacing at para-RBB may become another physiological pacing site other than His-bundle pacing, para-Hisian pacing and biventricular pacing.

\section{Conflict of interest: None declared}

\section{References}

1. Shimony A, Eisenberg MJ, Filion KB, Amit G. Beneficial effects of right ventricular non-apical vs. apical pacing: A systematic review and meta-analysis of randomized-controlled trials. Europace, 2012; 14: 81-91.

2. Muto C, Ottaviano L, Canciello $\mathrm{M}$ et al. Effect of pacing the right ventricular mid-septum tract in patients with permanent atrial fibrillation and low ejection fraction. J Cardiovasc Electrophysiol, 2007; 18: 1032-1036.

3. Mond HG, Hillock RJ, Stevenson IH, McGavigan AD. The right ventricular outflow tract: The road to septal pacing. Pacing Clin Electrophysiol, 2007; 30: 482-491.

4. Rosso R, Medi C, Teh AW, et al. Right ventricular septal pacing: A comparative study of outflow tract and mid ventricular sites. Pacing Clin Electrophysiol, 2010; 33: 1169-1173.

5. Kronborg MB, Poulsen SH, Mortensen PT, Nielsen JC. Left ventricular performance during para-His pacing in patients with high-grade atrioventricular block: An acute study. Europace, 2012; 14: 841-846.

6. Hillock RJ, Mond HG. Pacing the right ventricular outflow tract septum: Time to embrace the future. Europace, 2012; 14: 28-35.

7. Riedlbauchova L, Kautzner J, Hatala R, Buckingham TA. Is right ventricular outflow tract pacing an alternative to left ventricu- lar/biventricular pacing. Pacing Clin Electrophysiol, 2004; 27: 871-877.

8. Kappos KG, Tsagou V, Manolis AS. How harmful is conventional right ventricular apical pacing? Iatrogenic left bundle branch block: Need for alternate site pacing. Hospital Chronicles, 2006; supplement: 160-175.

9. Aksoy H, Okutucu S, Aytemir K et al. Baseline aortic pre-ejection interval predicts reverse remodeling and clinical improvement after cardiac resynchronization therapy. Cardiol J, 2011; 18: 639-647.

10. Lieberman R, Grenz D, Mond HG, Gammage MD. Selective site pacing: Defining and reaching the selected site. Pacing Clin Electrophysiol, 2004; 27: 883-886.

11. Lakshmanadoss U, Aggarwal A, Huang DT, Daubert JP, Shah A. Direct His bundle pacing post AVN ablation. Pacing Clin Electrophysiol, 2009; 32: 1101-1104.

12. Deshmukh PM, Romanyshyn M. Direct His-bundle pacing: Present and future. Pacing Clin Electrophysiol, 2004; 27: 862-870.

13. Kronborg MB, Mortensen PT, Gerdes JC, Jensen HK, Nielsen JC. His and para-His pacing in AV block: Feasibility and electrocardiographic findings. J Interv Card Electrophysiol, 2011; 31: 255-262.

14. Correa de Sa DD, Hardin NJ, Crespo EM, Nicholas KB, Lustgarten DL. Autopsy analysis of the implantation site of a permanent selective direct his bundle pacing lead. Circ Arrhythm Electrophysiol, 2012; 5: 244-246.

15. Lev M. Fine structure of the His bundle. Circulation, 1971; 44: 304-305.

16. Durrer D, van Dam RT, Freud GE, Janse MJ, Meijler FL, Arzbaecher RC. Total excitation of the isolated human heart. Circulation, 1970; 41: 899-912.

17. Mond HG, Hillock RJ, Stevenson IH, McGavigan AD. The right ventricular outflow tract: The road to septal pacing. Pacing Clin Electrophysiol, 2007; 30: 482-491.

18. Lazzara R, Yeh BK, Samet P. Functional transverse interconnections within the His bundle and the bundle branches. Circ Res, 1973; 32: 509-515. 